\title{
Single-cell Sequencing Resolves Landscape of Immune cell and Regulatory Mechanisms in HIV- infected immune non-responders
}

\section{Haiyu Li}

Chongqing Public Health Medical Center

\section{Yujing Wang}

Chongqing Medical University

\section{Yue Li}

Chongqing Medical University

Yi Yang

Chongqing Medical University

\section{Yuxin Xia}

Chongqing Medical University

Kui Liao

Chongqing Medical University

\section{Fangzhou Song}

Chongqing Medical University

\section{Shixiong Deng}

Chongqing Medical University

yaokai chen ( $\square$ lihaiyu@cqmu.edu.cn )

Chongqing Public Health Medical Center https://orcid.org/0000-0003-3403-9782

\section{Research Article}

Keywords: AIDS, single cell analysis, MAIT, mitochondrial dysfunction

Posted Date: December 14th, 2021

DOI: https://doi.org/10.21203/rs.3.rs-1140044/v1

License: (c) (i) This work is licensed under a Creative Commons Attribution 4.0 International License. Read Full License 


\section{Abstract}

Immune non-responder (INR) after highly active antiretroviral therapy (HAART) is the main cause of opportunistic infection and high mortality in AIDS patients, but the mechanism underlying immune reconstitution failure is poorly understood. Here we performed scRNA-seq and scATAC-seq analysis of peripheral blood mononuclear cells (PBMCs) derived from Immune nonresponder (INR) and responder (IR) HIV-1-infected subjects. We then describe the mitochondrial function of MAIT by flow cytometry in INRs and IRs. We founded there were diminished frequencies and numbers of MAIT cells in INRs, and MAIT cells from INRs displayed transcriptional profiles associated with the impairment of mitochondrial function and apoptosis signaling. ScATAC-seq and flow cytometry revealed diminished mitochondrial fitness in MAIT cells from INRs, and MAIT had low expression of transcription factor A for mitochondria (TFAM) and PPARA. These findings demonstrate the restoring mitochondrial function could modulate the immune dysfunction characteristic of MAIT, which against bacterial co-infections in INRs subjects.

\section{Introduction}

Acquired immune deficiency syndrome (AIDS) is a fatal infectious disease caused by human immunodeficiency virus (HIV). The main pathological feature is that the number of $\mathrm{CD} 4^{+}$lymphocytes is significantly reduced, resulting in a series of immunodeficiency related diseases. Combined antiretroviral therapy (CART) can significantly reduce the viral replication of HIV/AIDS patients, increase the number of $\mathrm{CD} 4^{+} \mathrm{T}$ lymphocytes, reconstitute the immune function of patients and reduce the mortality[1-5]. However, there are still $15-20 \%$ AIDS patients with complete inhibition of virus replication after cART, the number of $\mathrm{CD} 4^{+} \mathrm{T}$ cells still can not increase to the normal level of uninfected people, this part of patients is called immune non-response (INR) [6-10]. These INR subject is more likely to cause opportunistic infection or non AIDS related diseases, such as cardiovascular disease, liver and kidney disease, leading to increased mortality than immune responder (IR) subjects whom the $\mathrm{CD} 4^{+} \mathrm{T}$ cell count is reconstructed[11-15]. Opportunistic infections are common in INR patients, and the incidence rate and mortality are also high. In addition to AIDS related diseases and deaths, non AIDS related diseases and deaths are also higher in non immune responders[16-19]. Studies have confirmed that low $C D 4^{+} T$ cell counts increase the incidence rate and mortality of cardiovascular diseases, and are closely related to the occurrence of AIDS related or non related tumors and HIV related neurocognitive diseases.

To better understand the mechanism underlying immune reconstitution failure, we used single cell RNA sequencing (scRNA-seq) and Single Cell Assay for Transposase Accessible Chromatin with highthroughput sequencing (scATAC-seq) to examine the transcriptional profile of single cell from INRs, IRs. We founded there were diminished frequencies and numbers of mucosal associated invariant $\mathrm{T}$ cells (MAIT) in INRs, and MAIT cells from INRs displayed transcriptional profiles associated with the impairment of mitochondrial function and apoptosis signaling. MAITs are part of the immune system and their main task is to control bacteria on the body barrier (such as skin and mucosa). MAIT cells are a kind of unconventional T cells. They have the dual characteristics of innate and adaptive immunity. They 
can be activated in a TCR dependent or independent manner [20,21]. They have the ability to respond quickly to stimuli and can affect the response of T cells and B cells in the early stage of immune response [22-26]. In this study, we explore the molecular mechanism of MAIT mitochondrial dysfunction in HIV-infected immune nonresponders.

\section{Materials And Methods}

\section{Human blood samples}

Human blood samples involved in this study were approved by the Ethics Committee of Chongqing Public Health Medical Center. Informed consent was obtained from all each subjects.Immune nonresponder (INR) and responder (IR) HIV-1 infected patients were recruited at the AIDS Outpatient Department of Geleshan Hospital of Chongqing Public Health Medical Center. The blood samples of healthy individuals came from Chongqing Public Health Medical Center (Pingdingshan hospital area). PBMCs were isolated from fresh whole blood in $15 \mathrm{ml}$ centrifuge tube using Ficoll-Paque PREMIUM (Cytiva, USA, 17544203) according to the manufacturer's instructions. After mixing the blood sample with sterile PBS in equal volume, slowly add it to the Ficoll separation liquid level, and centrifuge $500 \mathrm{~g}$ horizontally for 15 minutes at room temperature. Cell numbers and viability were measured using hemocytometer with Trypan blue staining

\section{scRNA-seq library construction}

The single-cell library was constructed using the ChromiumTM Controller and Chromium Single Cell 5区 Library Kit. Briefly, single cells, reagents and Gel Beads containing barcoded oligonucleotides were encapsulated into nanoliter-sized GEMs using the GemCode Technology. Lysis and barcoded reverse transcription of polyadenylated mRNA from single cells was performed inside each GEM. Post RT-GEMs were cleaned up and cDNA were amplified. cDNA was fragmented and fragments end were repaired, as well A-tailing was added to the $3^{\prime}$ end. The adaptors were ligated to fragments which were double sided SPRI selected. Another double sided SPRI selecting was carried out after sample index PCR. The final library was quality and quantitated in two methods: check the distribution of the fragments size using the Agilent 2100 bioanalyzer, and quantify the library using real-time quantitative PCR (QPCR) (TaqMan Probe). The final products were sequenced using the Xten platform (BGI-Shenzhen, China).

\section{Single cell data analysis}

Cell Ranger Single Cell Software Suite (v3.1.0) was used to align complementary DNA reads to the reference genome. Single-cell FASTQ sequencing reads from each sample were processed, and converted to digital gene expression matrices. The dataset was trimmed of cells expressing fewer than 200 genes. The number of genes, UMI counts and percentage of mitochondrial genes were examined to identify outliers. Principal component analysis was used for dimensionality reduction, followed by clustering in principal component analysis space using a graph-based clustering approach. U-MAP was then used for two-dimensional visualization of the resulting clusters. For each clusters, the marker genes were identified 
using the FindConservedMarkers function as implemented in the Seurat package (logFC. threshold $>0.25$ and minPct>0.25). Then, clusters were remarked to a known cell type according to Cell Marker database [27-29]. Differently expressed genes across different samples were identified using the FindConservedMarkers function in Seurat by parameters of 'logFC. threshold $>0.25$, minPct $>0.25$ and Padj $\leq 0.05^{\prime}$. Pseudotime trajectory analysis was conducted with R package Monocle2[28].

\section{GO and KEGG analysis}

GO analysis and KEGG pathway analysis were performed using phyper, a function of R. Only GO terms or KEGG pathways with FDR $<=0.05$ were considered to be significant enrichment. WGCNA (v1.6.6) from $R$ package was used for identification of highly correlated gene by parameters of 'biweight midcorrelation> $0.7^{\prime}$, highly correlated gene were used for final correlation network construction.

\section{Antibodies and Flow cytometry analysis}

Fluorochrome-conjugated monoclonal antibodies specific for human antigens were used anti-CD4-FITC (BD Bioscience, catalog 561005), anti-TCR Va7.2-APC (BioLegend, catalog 351708), anti-IFN-Y-APC (BD Bioscience, catalog 562017), Anti-CD3-FITC (Bioscience, catalog 555339), Anti-CD161- PE(BD Bioscience, catalog 556081). Intracellular staining was performed using eBioscience ${ }^{\mathrm{TM}}$ Intracellular Fixation \& Permeabilization Buffer Set (ThermoFisher) according to the manufacturer's protocols.

\section{Cytokine antibody microarray assay}

The AAH-BLG-507 cytokine antibody microarray (Raybiotech, Norcross, GA, USA) were used to detect cytokines in plasma samples derived from IR and INR subjects. The detection of cytokines was performed according to the manufacturer's instructions. Briefly, plasma is subjected to sample dialysis, sample labeling, antibody array blocking and incubation, antibody array scanning detection. When the raw data are analyzed, the background removed fluorescent signal FI (F532 medium-B532 medium) is generally used for analysis, the mean value of two repetitions of all antibodies is calculated, and the mean value is used as the signal value of each antibody for subsequent analysis. All antibodies of each sample were normalized with all positive controls to obtain the normalized signal value. Generally, in the chip experiment results, for proteins with low signal value, the signal value $\mathrm{fl}<25$ is regarded as not detected.

\section{Luminex liquid suspension microarray}

The concentrations of selected cytokines including IF1 the plasma samples derived from 51 IR subjects and 24 INR subjects were detected using human high sensitivity T cell magnetic bead panel (Milipore,) according to the manufacturer's instructions.

The samples generally go through the steps of incubation of samples with magnetic beads, incubation of detection antibodies and Luminex200 detection. After the samples and standards tested in this experiment are detected by Luminex 200 detector, the fluorescence obtained is automatically detected 
and optimized by software to form the raw data. According to the fluorescence detection value obtained from the standard, the standard curve and its equation are obtained by fitting the standard curve with multi parameter mode, and the concentration unit is $\mathrm{pg} / \mathrm{ml}$. The original fluorescence detected by each sample is substituted into the standard curve formula to calculate the sample concentration, which is used for comparison between samples.

\section{Results}

\section{Single-cell transcriptome altas of PBMCs in IRs and INRs}

To identify the immunological features of HIV-1-infected patients, we performed droplet based single-cell RNA sequencing technology (10×Genomics) of fresh PBMCs derived from 2 immune nonresponder (INR1,INR2) and 2 immune responder controls (IR1,IR2). After quality control and unified single-cell analysis pipeline, The total number of recovered cells was 12086 comprising 5172 cells for INR and 6915 cells for controls IRs detecting a mean of 1,445 and 1,384 genes per cell, respectively (Fig. 1A,B and Fig.S1A). We calculated subsets that showed high differences between cells in the data set (i.e., they were highly expressed in some cells and low expressed in others). The screened differential genes with hypervariable characteristics will be used for downstream principal component analysis. We visualized the cells in two dimensional spaces according to their expression profiles using graph-based clustering of uniform manifold approximation and projection (UMAP), a nonlinear dimensionality reduction algorithm (Fig.1A). Based on the expression of canonical gene markers[29], we identified cells to 11 major cell types or subtypes: CD8 effector T, NK cell,T helper,B cell, classical monocytes, non classical monocytes, pDCs, mucosal-associated invariant T cell, platelets, CD4 naïve T,CD8 naïve T (Fig.1B). List of marker genes defining cell subsets are detailed in Supplementary Table 1 and fig.S1B.To visual display of single-cell RNA-Seq data, we constructed a website at http://47.94.11.173:8081/\#/data. This website is completely open and can view expression information of genes of interest in specific cell types without registration. These clusters and their specific markers included CD3D, CD8A, CD8B, NKG7 etc (Fig.1C, D).

\section{Significant reduction and dysfunction of MAIT cells in INRs}

Single cell transcriptome sequencing showed that the proportion of MAIT cells in INR decreased significantly. To demonstrate the frequency of MAIT cells, FACS analyses were performed in peripheral blood mononuclear cells (PBMCs) from 17 Immune nonresponder (INR) HIV-1-infected subjects, 10 Immune responder (INR) HIV-1-infected subjects, 10 uninfected healthy blood donors. MAIT cells were identified using cell-surface markers $C D 3^{+}$TCRV7. $2^{+}$CD $161^{\text {high }}$ in PBMCs (Fig.2A). We found that the frequency of MAIT cells was significantly lower in INRs compared with frequencies in the IRs or HCs (Fig.2B). INRs were defined as having $\mathrm{CD}^{+} \mathrm{T}$ cell counts below $350 \mathrm{cells} / \mu \mathrm{l}$ and IRs as having $\mathrm{CD} 4+\mathrm{T}$ cells counts above $350 / \mu \mathrm{l}$ after at least 2 years of cART with virologic control. The level of IFN gamma was detected in MAIT cells derived from IR, INR and HC stimulated by paraformaldehyde (PFA)-fixed $E$. coli. MAIT cells from INRs produced lower levels of IFN gamma, in response to $E$. coli stimulation as 
compared to the HC group (Fig.2C, D). These results suggest that MAIT derived from INR has dysfunction in resisting infection by other pathogens.

\section{MAIT cells from INRs have distinct gene expression profiles reflecting apoptotic signaling and inflammatory responses}

To define the molecular mechanism of MAIT dysfunction in INRs, we next explored differences in gene expression profiles of MAIT cells between INRs and IRs. We founded that the expression of proapoptotic genes in MAIT cells was significantly $(P<0.05)$ upregulated specifically in the INRs when compared with expression of these genes in IRs (Fig.3A). Furthermore, we also funded that the expression of proapoptotic gene IFI27, IFIT2, SAMD9 was significantly increased in MAIT cells from INRs (Fig.3B). As shown in figure3C, the expression of $C D 54, K L R B 1$, and $J U N$, which the regulator of $\mathrm{T}$ cell function $\nabla$ and MT-ND1,MT-ATP6 and RPS26, which the master regulator of mitochondrial biogenesis significantly diminished expressed in MAIT cells from INRs. It is known that mitochondrial function is closely related to apoptosis [30-32]. We analyzed the transcriptional profile of mitochondrial related genes (MT-ND1, MTATP6 and RPS26) in INR and found decreased expression of genes involved in different phases of mitochondrial function of MAIT cells from INRs. In order to confirm whether the mitochondrial function of MAIT is impaired, Mito-Tracker Green (MG) and Mito-Tracker Red CMXRos (MRC) were used to detect mitochondrial mass and mitochondrial membrane potential, respectively (Fig.3D). The oxidative phosphorylation activity measured by the mean fluorescence intensity of MCR was significantly decreased in MAIT cells derived from INRs. Similarly, when the mean fluorescence intensity of MG was used to measure the quality of mitochondria, the MFI significantly decreased in MAIT cell from INR (Fig.3D). The Gene Ontology (GO) and KEGG enrichment analysis further showed that the upregulated genes in MAIT cells from INRs were enriched in Type I IFN and apoptosis pathways (Fig.3E, F).

\section{TFAM and PPARA low expression was associated with MAIT cell dysfunction of INRs}

To identify the mechanism that drives MAIT cell dysfunction, we performed scATAC-Seq on PBMC derived from INR. After normalization and dimensional reduction, we identified nine major clusters of cells, together with open chromatin peaks specific to each cluster (Fig.4A). We integrated the scRNAseq and scATACseq datasets to correlate and cross-validate gene expression profiles and chromatin accessibility landscape in INR (Fig.4B). To identify transcription factor (TFs) that are essential for cell-type-specific gene expression, we profiled TF binding motifs overrepresented in the peaks that showed differential accessibility among cell types (Fig.4C Fig.S2A). We also found that the chromatin of BCL11B was active and open in MAIT cells derived from INR, indicating that BCL11B may be highly expressed in MAIT cells derived from INR (Fig.4D). Having established the high concordance between the scRNAseq and scATACseq data, we founded that transcription factor A for mitochondria (TFAM) binding activity abruptly decreased in INR (Fig.4E, Fig.S2B). Furthermore, the expression of TFAM and peroxisomal proliferator-activated receptor alpha (PPARA) the master regulator of mitochondrial biogenesis were detected by flow cytometry. As shown in figure4F, MAIT cells from INRs showed significantly decreased expression of PPARA and TFAM. 


\section{Significant reduction of cytotoxic CD4 T cells in INRs}

The previous single cell analysis showed that the proportion of Th cells in INR decreased significantly, and Th cells played an important role in the immune system. Their function was to produce a variety of cytokines, transmit antigen information, promote the differentiation and proliferation of $\mathrm{T}$ and $\mathrm{B}$ cells, and assist $B$ cells to produce antibodies. To better understand the function of this Th cell specific population in INR, Th cells were extracted from all cells and further analyzed using Seurat R software package. Th cluster was further divided into four small cell subsets using a clustering algorithm based on shared nearest neighbor modular optimization (Fig.5A). Based on the expression of established marker genes, we identified a marked increase of cytotoxic CD4 T cells (CD4 cytotoxic T lymphocytes [CTLs]) in IR and INR group (Fig.5A). We mapped CD4 CTL to the two-dimensional UMAP of single cell transcriptome and found that the proportion of CD4 CTL in INR was significantly reduced compared with IR (Fig.5B).We then compared these 2 groups and identified 440 differentially expressed genes of CD4 CTL, Go enrichment analysis of highly expressed genes in CD4 CTL derived from INR showed that it was cell over activation (Fig.5B).

\section{The profile of CD8 effector T in INRs}

CD8 effector T cells are specific T cells that secrete various cytokines to participate in immune function. They can kill some antigenic substances such as viruses and tumor cells. Our previous results showed that there was no significant difference in the proportion of CD8 effector T between INR and IR. In order to better understand the function of CD8 effector T, we extracted all of the cells and subdivided them into 11 small cell clusters (C1-11) using the Seurat R package (Fig.6A, B). The proportion of CD8 effector T C1 and $\mathrm{C} 2$ is significantly different in INR and IR, which may be due to different biological functions (Fig.6C). We then compared these 2 groups and identified differentially expressed genes of CD8 effector T, CD8 effector T C1 and C2 (Fig.6D, E and F). Go enrichment analysis of highly expressed genes in CD8 effector T $\mathrm{C} 1$ derived from INR showed that it was involved in type 1 interferon signaling pathway (Fig.6G).

\section{Cell State Transition of CD8 effector T, MAIT and CD4 CTL during T Cell Differentiation}

To understand the difference of T cell differentiation in INR and IR, we constructed single-cell trajectories using the Monocle2 $\mathrm{R}$ package. According to the changes of gene expression profile, all $\mathrm{T}$ cells in CD8 effector T, MAIT and CD4 CTL were placed on these trajectories (Fig.7A). Consistent with the clustering analyses, there was continuity in the differentiation of MAIT and Th cells in IRs, while there was a gap in the differentiation trajectory in INRs (Fig.7B). There was no significant difference in the differentiation trajectories of CD8 effector T cells in IR and INR (Fig.7C).

\section{Identification of cytokine profile for INR and IR}

In toeder to screening differential cytokines between INR and IR, L-serious 507 antibody-based protein microarrays were performed to measure the inflammatory cytokine expression profile. Expression levels 
of 62 cytokines were significantly different between the INR and IR patients $(P<0.05)$. The top 20 cytokines with the most significant differences are shown in Figure 8A. To verify the results of the cytokine microarray, liquid suspension cytokine microarray was performed to detect concentrations of IL4, MCP-1, IL-7 and IL-15 in plasma samples from 51 IR subjects and 24 INR subjects (Fig.8B, C, D, E). The GO and KEGG enrichment analysis further showed that the up-regulated cytokine in plasma from INR subjects were enriched in cellular activation pathways including cytokine-cytokine receptor interaction, inflammatory bowel disease etc, indicating that INRs had abnormal immune activation (Fig.8F).

\section{Discussion}

Highly active antiretroviral therapy (HAART) can significantly reduce the virus replication of HIV/AIDS patients, increase the number of CD4 + T lymphocytes and reconstruct the immune function of patients [33]. However, there are still some patients with immune reconstitution failure and immune reconstitution failure under the condition of virus inhibition[34]. There are many factors related to the failure of immune reconstitution in HIV/AIDS patients. Previous studies have conducted retrospective studies on patients with poor immune reconstitution. It is found that age, baseline $\mathrm{CD} 4^{+} \mathrm{T}$ lymphocyte level, HCV co infection and other factors can affect the immune reconstitution of patients[35-38]. However, the mechanism underlying immune reconstitution failure is poorly understood. Single cell sequencing technology is a new technology for high-throughput sequencing analysis of genome, transcriptome and epigenome at the single cell level. It can reveal the gene structure and gene expression state of a single cell, reflect the heterogeneity between cells, and can well reveal the biological mechanism of disease.

In this study, Firstly, we sequenced the single-cell transcriptome of PBMC from patients with INR and IR. Cluster analysis identified 11 cell subsets, including CD8 effector T, NK cell,T helper,B cell, classical monocytes, non classical monocytes, pDCs, mucosal-associated invariant T cell, platelets, CD4 naïve T,CD8 naïve T. Single cell transcriptome sequencing and flow cytometry results confirmed that the proportion of MAIT cells in INR decreased significantly compared with IR subjects. We also found that MAIT cells derived from INR had dysfunction under the stimulation of $E$. coli and could not produce interferon response. MAIT cells derived from INR are in a state of depletion, resulting in dysfunction. About $5 \%$ of T cells in healthy people's blood are composed of MAIT cells. They are mainly used to control bacteria, but they can also be recruited by the immune system to fight some viral infections. The results of scATAC-seq and flow cytometry showed that the low expression of TFAM and PPARA was the main cause of mitochondrial dysfunction in MAIT cells derived from INR.

Furthermore, we identified a marked increase of cytotoxic CD4 T cells (CD4 cytotoxic T lymphocytes [CTLs]) in IR and INR subjects. In 1977, it was first reported that CD4 T cells cultured in vitro have the characteristics of cytotoxicity. There are few studies on CD4 T cytotoxic cells, and there has been a dispute about whether CD4 T CTL exists in human body. In 2001, sunl et al reported that CD $4^{+}$CD $^{-}{ }^{-} T$ lymphocytes had strong cytokine expression and proliferation ability and showed the function of cytotoxic activity when infected with HCV and HIV-1 virus. Since then, the research on CD4 cytotoxic T cells has gradually attracted attention. In this study, we also found a cluster of $C D 4^{+} T$ lymphocytes with 
high specific expression of cytotoxicity related genes, which further confirmed that there may be CD $4^{+}$ CTL in human body and may have certain cytotoxicity related functions. Therefore, this cluster is defined as "CD4 ${ }^{+}$CTL".

In summary, we revealed the single-cell expression profiles in INR and IR by SCRNA-seq and ScATAC, and confirmed the main cause of mitochondrial dysfunction caused by low expression of TFAM in MAIT derived from INR. This is linked to poor clinical outcome.

\section{Abbreviations}

INR (Immune non-responder); IR (Immune responder); KEGG (Kyoto Encyclopedia of Genes and Genomes); GO (Gene Ontology); scATAC(single cell Assay for Transposase-Accessible Chromatin); UMI (Unique molecular identifier); TCR (T cell receptor); BCR (B cell receptor)

\section{Declarations}

\section{Acknowledgements}

This work was supported by the National Natural Science Foundation of China [No.82002154], National Science and Technology Major Project of China during the 13th Five-year plan period [No.

2018ZX10302104] and the General Project of Chongqing Basic Research and Frontier Exploration Project (Natural Science Foundation), China [No. cstc2019jcyj-msxmX0029].

\section{Declaration of interest statement}

The authors declare that they have no competing interests.

\section{Authors' contributions}

Haiyu Li, Kui Liao*,Fangzhou Song, Shixiong Deng and Yaokai Chen performed the experiment, were major contributors in writing and submitting the manuscript and drafted the manuscript. Yujing Wang, Yue Li retrieved the data and performed the statistical analysis. Yi Yang, Yuxin Xia, Li Rong, Shengquan Tang performed the bioinformatics analysis. All authors read and approved the final manuscript.

\section{Availability of data and materials}

All data generated and analyzed during this study are included in this published article and its additional files.

\section{Consent for publication}

Not applicable 
The study was approved by the ethical committee of Chongqing Public Health Medical Center, China.

\section{References}

1. Grinsztejn B, Hosseinipour M, Ribaudo H, Swindells S, Eron J, Chen Y, Wang L, Ou S, Anderson M, McCauley $M$ et al: Effects of early versus delayed initiation of antiretroviral treatment on clinical outcomes of HIV-1 infection: results from the phase 3 HPTN 052 randomised controlled trial. The Lancet Infectious diseases 2014, 14(4):281-290.

2. Lundgren J, Babiker A, Gordin F, Emery S, Grund B, Sharma S, Avihingsanon A, Cooper D, Fätkenheuer G, Llibre J et al: Initiation of Antiretroviral Therapy in Early Asymptomatic HIV Infection. The New England journal of medicine 2015, 373(9):795-807.

3. Pierre S, Jannat-Khah D, Fitzgerald D, Pape J, McNairy M: 10-Year Survival of Patients with AIDS Receiving Antiretroviral Therapy in Haiti. The New England journal of medicine 2016, 374(4):397-398.

4. Melgar M, Nichols C, Cavanaugh J, Kirking H, Surie D, Date A, Ahmedov S, Maloney S, Fukunaga R: Tuberculosis Preventive Treatment Scale-Up Among Antiretroviral Therapy Patients - 16 Countries Supported by the U.S. President's Emergency Plan for AIDS Relief, 2017-2019. MMWR Morbidity and mortality weekly report 2020, 69(12):329-334.

5. Ganatra S, Bucşan A, Alvarez X, Kumar S, Chatterjee A, Quezada M, Fish A, Singh D, Singh B, Sharan $\mathrm{R}$ et al: Antiretroviral therapy does not reduce tuberculosis reactivation in a tuberculosis-HIV coinfection model. The Journal of clinical investigation 2020, 130(10):5171-5179.

6. Guihot A, Bourgarit A, Carcelain G, Autran B: Immune reconstitution after a decade of combined antiretroviral therapies for human immunodeficiency virus. Trends in immunology 2011, 32(3):131-137.

7. Autran B, Carcelaint G, Li TS, Gorochov G, Blanc C, Renaud M, Durali M, Mathez D, Calvez V, Leibowitch $\mathrm{J}$ et al: Restoration of the immune system with anti-retroviral therapy. Immunology letters 1999, 66(1-3):207-211.

8. Grabar S, Le Moing V, Goujard C, Leport C, Kazatchkine M, Costagliola D, Weiss L: Clinical outcome of patients with HIV-1 infection according to immunologic and virologic response after 6 months of highly active antiretroviral therapy. Annals of internal medicine 2000, 133(6):401-410.

9. Yang $X$, Su B, Zhang $X$, Liu Y, Wu H, Zhang T: Incomplete immune reconstitution in HIV/AIDS patients on antiretroviral therapy: Challenges of immunological non-responders. Journal of leukocyte biology 2020, 107(4):597-612.

10. Powderly W, Landay A, Lederman M: Recovery of the immune system with antiretroviral therapy: the end of opportunism? JAMA 1998, 280(1):72-77. 
11. Engsig F, Gerstoft J, Kronborg G, Larsen C, Pedersen G, Røge B, Jensen J, Nielsen L, Obel N: Longterm mortality in HIV patients virally suppressed for more than three years with incomplete CD4 recovery: a cohort study. BMC infectious diseases 2010, 10:318.

12. Monforte A, Abrams D, Pradier C, Weber R, Reiss P, Bonnet F, Kirk O, Law M, De Wit S, Friis-Møller $\mathrm{N}$ et al: HIV-induced immunodeficiency and mortality from AIDS-defining and non-AIDS-defining malignancies. AIDS (London, England) 2008, 22(16):2143-2153.

13. Bowen L, Smith B, Reich D, Quezado M, Nath A: HIV-associated opportunistic CNS infections: pathophysiology, diagnosis and treatment. Nature reviews Neurology 2016, 12(11):662-674.

14. Tan I, Smith B, von Geldern G, Mateen F, McArthur J: HIV-associated opportunistic infections of the CNS. The Lancet Neurology 2012, 11(7):605-617.

15. Picat M, Lewis J, Musiime V, Prendergast A, Nathoo K, Kekitiinwa A, Nahirya Ntege P, Gibb D, Thiebaut $\mathrm{R}$, Walker $\mathrm{A}$ et al: Predicting patterns of long-term CD4 reconstitution in HIV-infected children starting antiretroviral therapy in sub-Saharan Africa: a cohort-based modelling study. PLOS medicine 2013, 10(10):e1001542.

16. Cribbs S, Crothers K, Morris A: Pathogenesis of HIV-Related Lung Disease: Immunity, Infection, and Inflammation. Physiological reviews 2020, 100(2):603-632.

17. Baker J, Peng G, Rapkin J, Abrams D, Silverberg M, MacArthur R, Cavert W, Henry W, Neaton J: CD4+ count and risk of non-AIDS diseases following initial treatment for HIV infection. AIDS (London, England) 2008, 22(7):841-848.

18. Kelley C, Kitchen C, Hunt P, Rodriguez B, Hecht F, Kitahata M, Crane H, Willig J, Mugavero M, Saag $\mathrm{M}$ et al: Incomplete peripheral CD4+ cell count restoration in HIV-infected patients receiving long-term antiretroviral treatment. Clinical infectious diseases : an official publication of the Infectious Diseases Society of America 2009, 48(6):787-794.

19. Shive C, Mudd J, Funderburg N, Sieg S, Kyi B, Bazdar D, Mangioni D, Gori A, Jacobson J, Brooks A et al: Inflammatory cytokines drive CD4+ T-cell cycling and impaired responsiveness to interleukin 7: implications for immune failure in HIV disease. The Journal of infectious diseases 2014, 210(4):619-629.

20. Awad W, Ler G, Xu W, Keller A, Mak J, Lim X, Liu L, Eckle S, Le Nours J, McCluskey J et al: The molecular basis underpinning the potency and specificity of MAIT cell antigens. Nature immunology 2020, 21(4):400-411.

21. Le Bourhis L, Martin E, Péguillet I, Guihot A, Froux N, Coré M, Lévy E, Dusseaux M, Meyssonnier V, Premel $\mathrm{V}$ et al: Antimicrobial activity of mucosal-associated invariant T cells. Nature immunology 2010, 11(8):701-708. 
22. Toubal A, Nel I, Lotersztajn S, Lehuen A: Mucosal-associated invariant T cells and disease. Nature reviews Immunology 2019, 19(10):643-657.

23. Legoux F, Bellet D, Daviaud C, El Morr Y, Darbois A, Niort K, Procopio E, Salou M, Gilet J, Ryffel B et al: Microbial metabolites control the thymic development of mucosal-associated invariant $\mathrm{T}$ cells. Science (New York, NY) 2019, 366(6464):494-499.

24. Koay H, Gherardin N, Enders A, Loh L, Mackay L, Almeida C, Russ B, Nold-Petry C, Nold M, Bedoui $\mathrm{S}$ et al: A three-stage intrathymic development pathway for the mucosal-associated invariant $\mathrm{T}$ cell lineage. Nature immunology 2016, 17(11):1300-1311.

25. Jeffery H, van Wilgenburg B, Kurioka A, Parekh K, Stirling K, Roberts S, Dutton E, Hunter S, Geh D, Braitch $M$ et al: Biliary epithelium and liver B cells exposed to bacteria activate intrahepatic MAIT cells through MR1. Journal of hepatology 2016, 64(5):1118-1127.

26. Legoux F, Salou M, Lantz O: MAIT Cell Development and Functions: the Microbial Connection. Immunity 2020, 53(4):710-723.

27. Butler A, Hoffman P, Smibert P, Papalexi E, Satija R: Integrating single-cell transcriptomic data across different conditions, technologies, and species. Nature biotechnology 2018, 36(5):411-420.

28. Qiu X, Hill A, Packer J, Lin D, Ma Y, Trapnell C: Single-cell mRNA quantification and differential analysis with Census. Nature methods 2017, 14(3):309-315.

29. Zhang X, Lan Y, Xu J, Quan F, Zhao E, Deng C, Luo T, Xu L, Liao G, Yan M et al: CellMarker: a manually curated resource of cell markers in human and mouse. Nucleic acids research 2019, 47:D721D728.

30. Milasta S, Dillon C, Sturm O, Verbist K, Brewer T, Quarato G, Brown S, Frase S, Janke L, Perry S et al: Apoptosis-Inducing-Factor-Dependent Mitochondrial Function Is Required for T Cell but Not B Cell Function. Immunity 2016, 44(1):88-102.

31. Bock F, Tait S: Mitochondria as multifaceted regulators of cell death. Nature reviews Molecular cell biology 2020, 21(2):85-100.

32. Liang J, Cao R, Wang X, Zhang Y, Wang P, Gao H, Li C, Yang F, Zeng R, Wei P et al: Mitochondrial PKM2 regulates oxidative stress-induced apoptosis by stabilizing Bcl2. Cell research 2017, 27(3):329-351.

33. Katz I, Leister E, Kacanek D, Hughes M, Bardeguez A, Livingston E, Stek A, Shapiro D, Tuomala R: Factors associated with lack of viral suppression at delivery among highly active antiretroviral therapynaive women with HIV: a cohort study. Annals of internal medicine 2015, 162(2):90-99.

34. Lewden C, Chene G, Morlat P, Raffi F, Dupon M, Dellamonica P, Pellegrin JL, Katlama C, Dabis F, Leport C: HIV-Infected Adults With a CD4 Cell Count Greater Than 500 Cells/mm^3 on Long-Term 
Combination Antiretroviral Therapy Reach Same Mortality Rates as the General Population. JAIDS HAGERSTOWN MD- 2007.

35. Ahuja S, Kulkarni H, Catano G, Agan B, Camargo J, He W, O'Connell R, Marconi V, Delmar J, Eron $\mathrm{J}$ et al: CCL3L1-CCR5 genotype influences durability of immune recovery during antiretroviral therapy of HIV-1-infected individuals. Nature medicine 2008, 14(4):413-420.

36. Younes S, Talla A, Pereira Ribeiro S, Saidakova E, Korolevskaya L, Shmagel K, Shive C, Freeman M, Panigrahi S, Zweig S et al: Cycling CD4+ T cells in HIV-infected immune nonresponders have mitochondrial dysfunction. The Journal of clinical investigation 2018, 128(11):5083-5094.

37. Scherpenisse M, Kootstra N, Bakker M, Berkhout B, Pasternak A: Cell-Associated HIV-1 Unsplicedto-Multiply-Spliced RNA Ratio at 12 Weeks of ART Predicts Immune Reconstitution on Therapy. mBio 2021, 12(2).

38. HIV Infection Masquerading as Monoclonal Gammopathy of Unknown Significance. N Engl J Med 2003, 349(24):2362-2363.

\section{Supplementary Table}

Supplementary Table 1 is not available with this version.

\section{Figures}



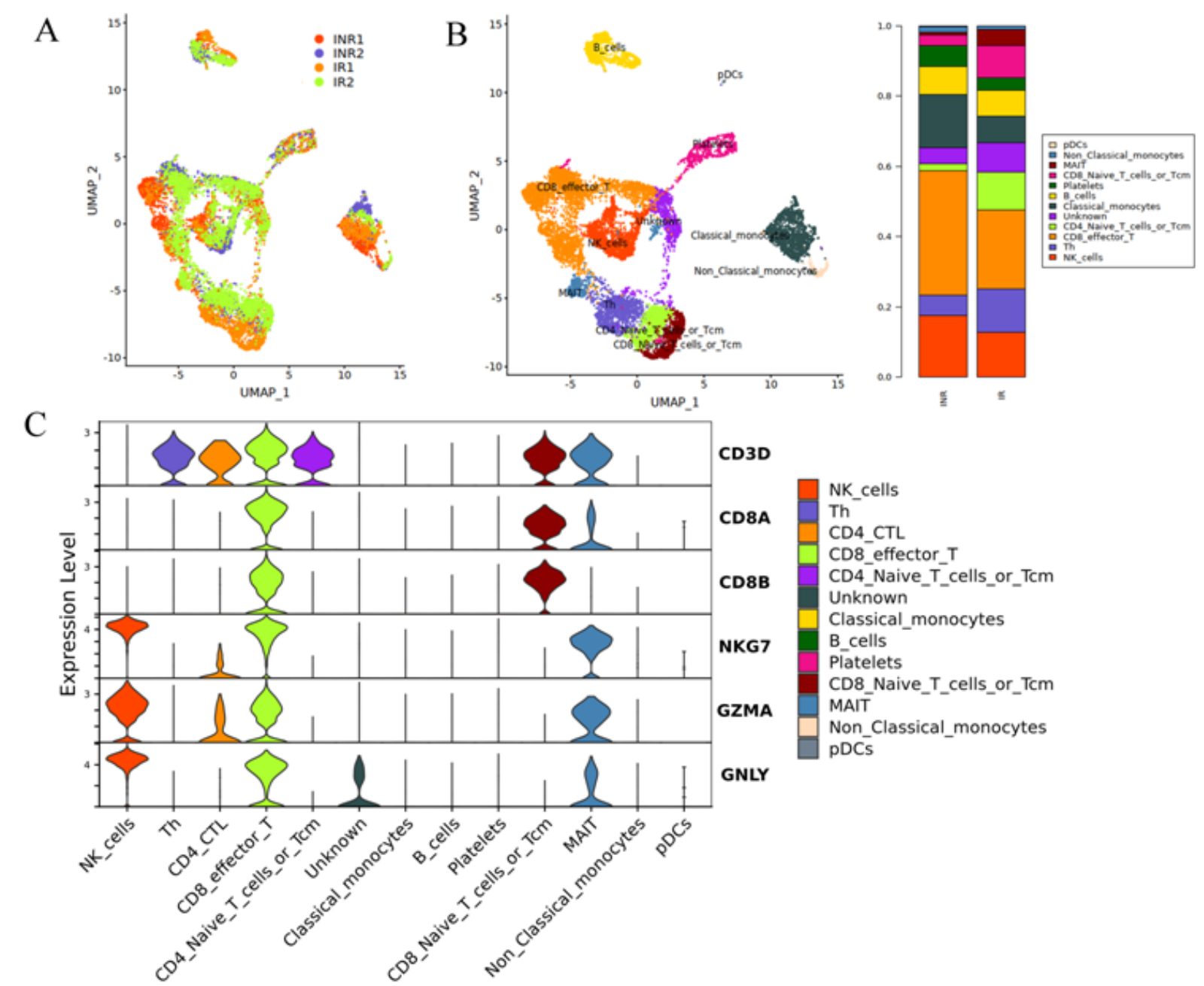

$\mathrm{D}$

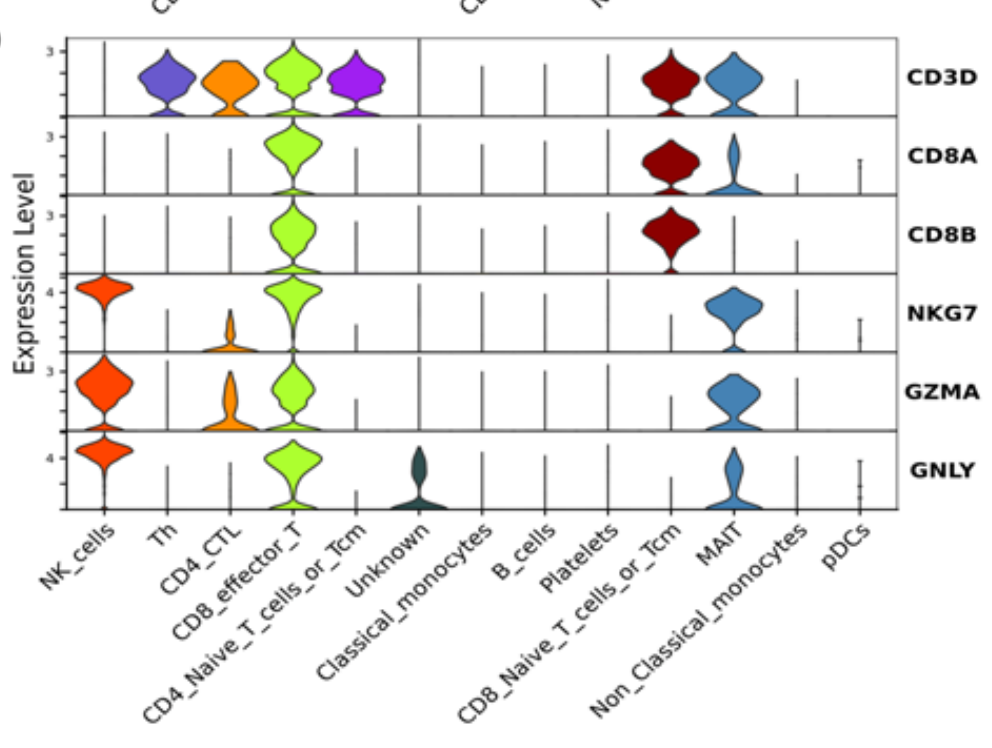

NK_cells

Th

CD4_CTL

CD8 effector T

CD4_Naive_T_cells_or_Tcm

Unknown

Classical_monocytes

B_cells

Platelets

CD8_Naive_T_cells_or_Tcm

MAIT

Non_Classical_monocytes

pDCs

.

\section{Figure 1}

single-cell transcriptional profiling of PBMCs derived from IR and INR patients with HIV-1-infected. (A) Two-dimensional UMAP visualization of PBMCs for IRs and INRs. Different colors represent different sample sources. (B) Different colors represent 10 clusters (cell types) defined by the k-means clustering algorithm. Different colors represent 11cell types defined. The UMAP projection of 12086 single cells from IR and INR samples, showing the formation of 11 clusters with the respective labels. Each dot 
corresponds to a single cell, colored according to cell type. (C, D) Stacked violin plots showing the expression distribution of selected canonical cell markers in the MAIT CD8 effector T
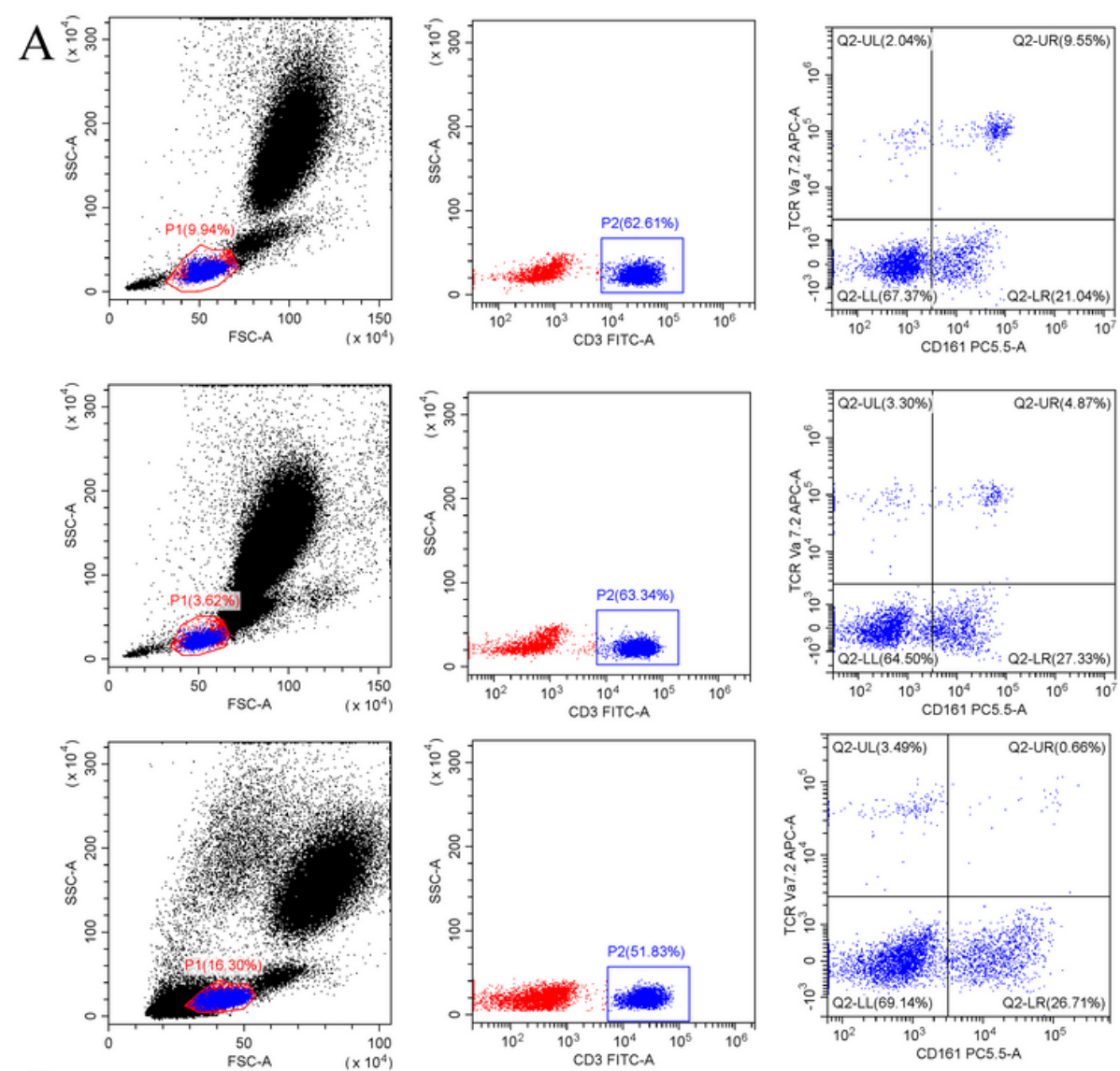

$\mathrm{C}$
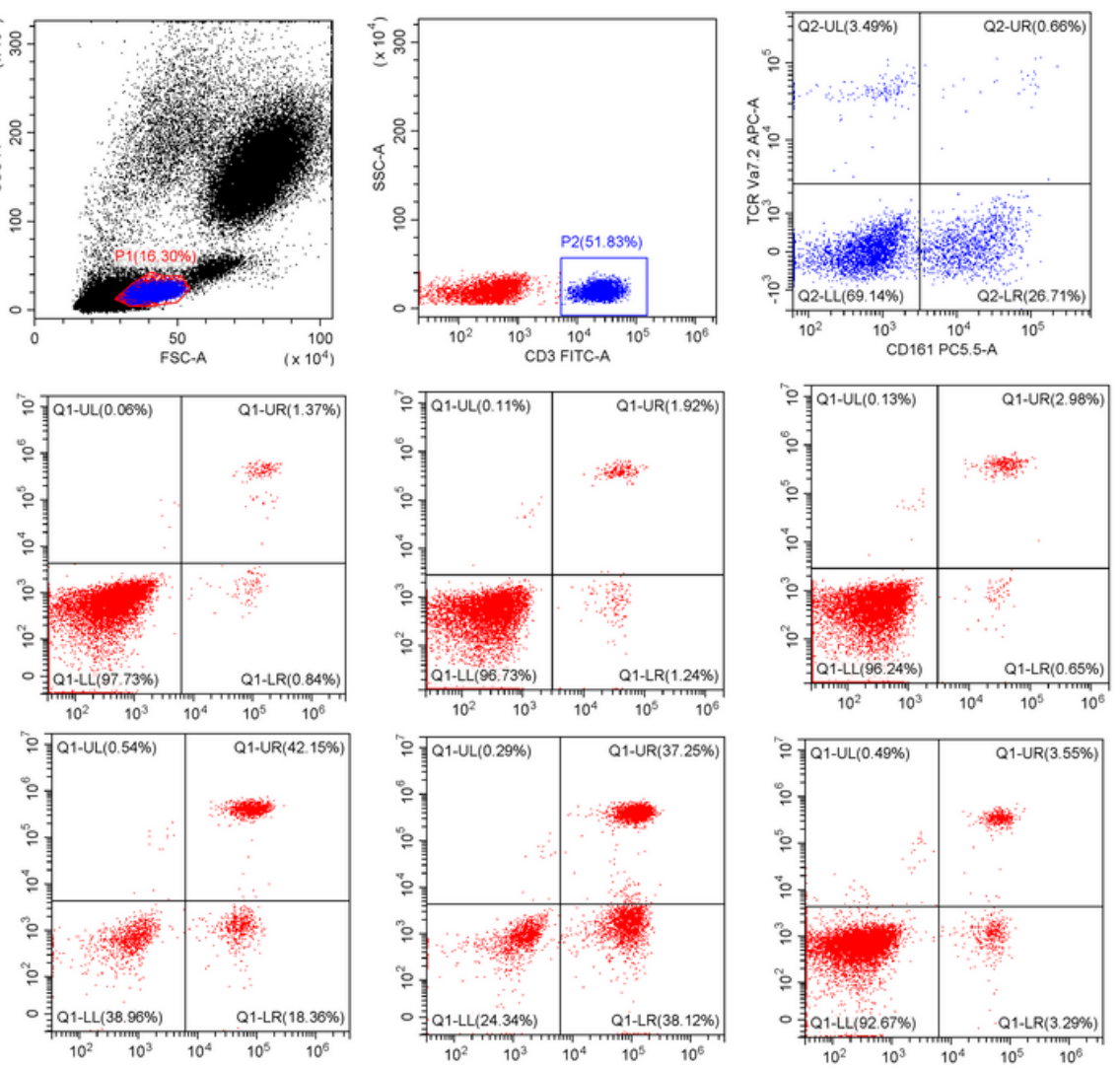
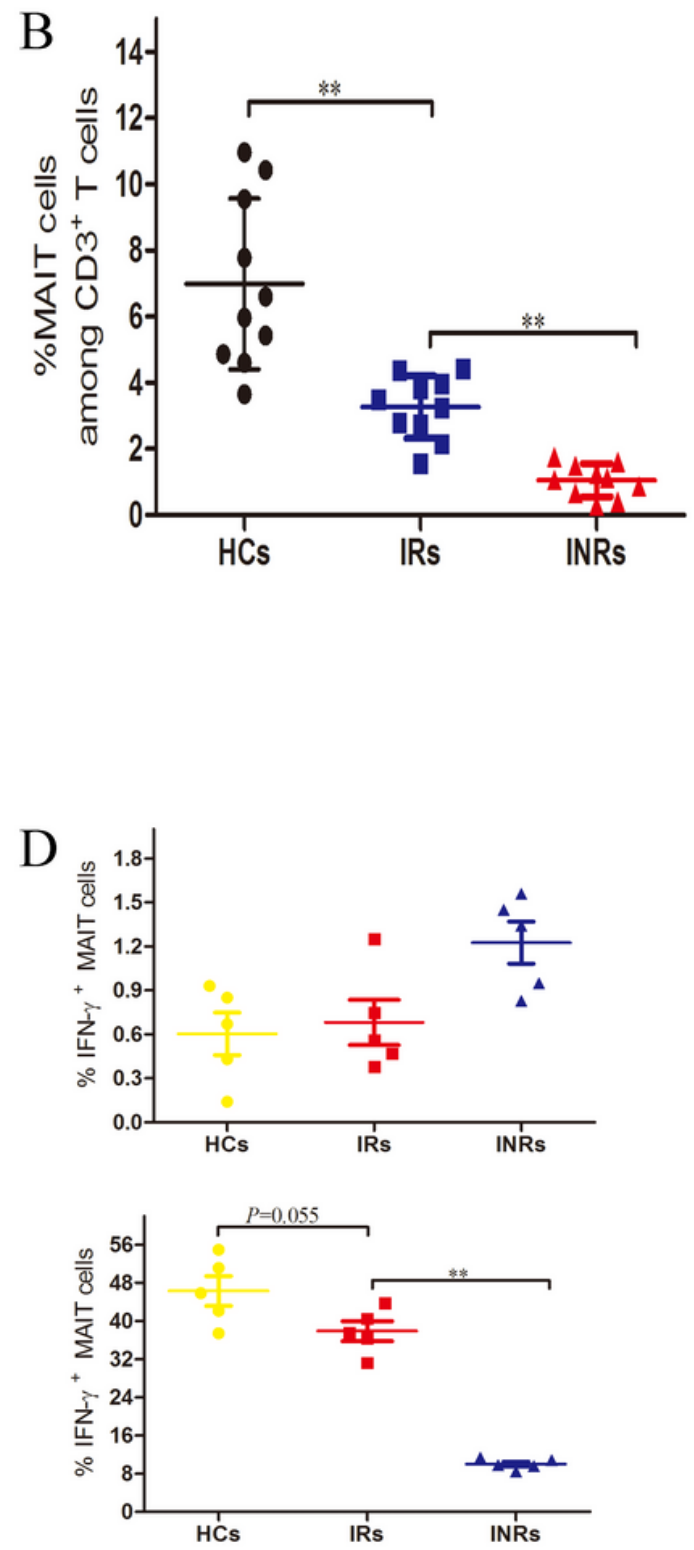

Figure 2

MAIT derived from INR is dysfunctional. (A, B)Proportions of MAIT cells were detected in PBMCs from health control (HC), immune nonresponder (INR) HIV-1-infected subjects and immune responder (IR) HIV1-infected subjects. Representative FC (flow cytometry) plots (A) and statistical analysis (B) are shown. (C, D) Detection of interferon gamma produced by MAIT after PBMC isolated from HCs, INRs and IRs was stimulated by paraformaldehyde (PFA)-fixed E.coli. 
A

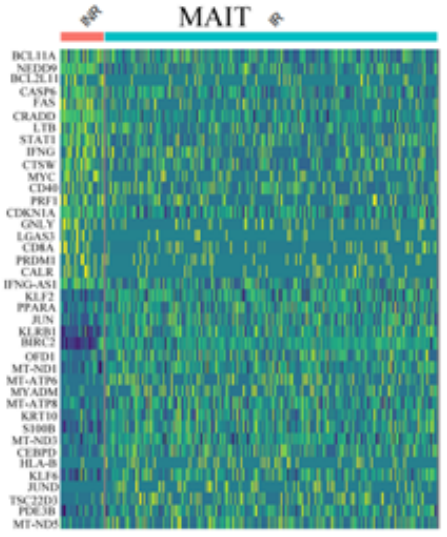

$\mathrm{C}$
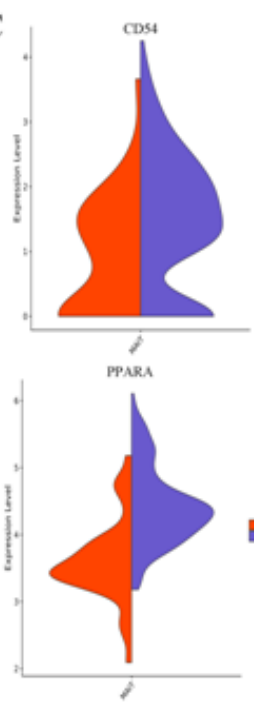

B

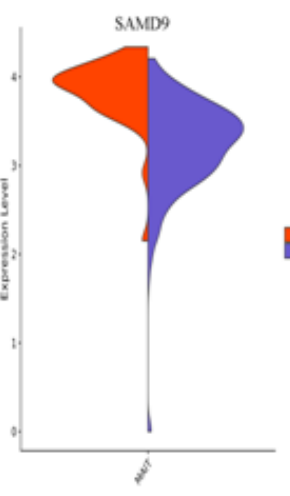

$I *$
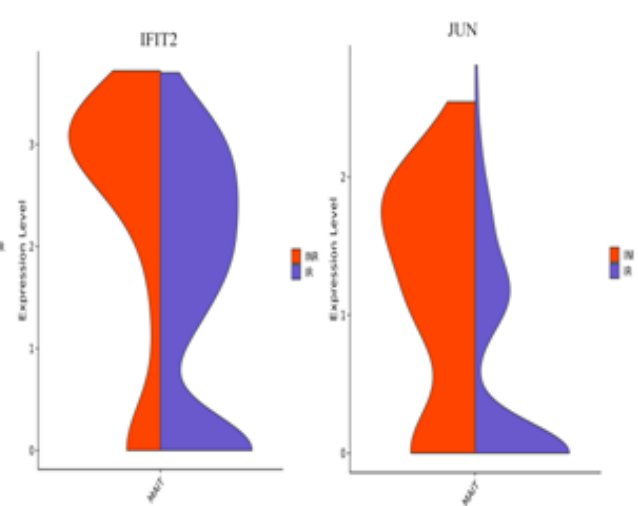

D
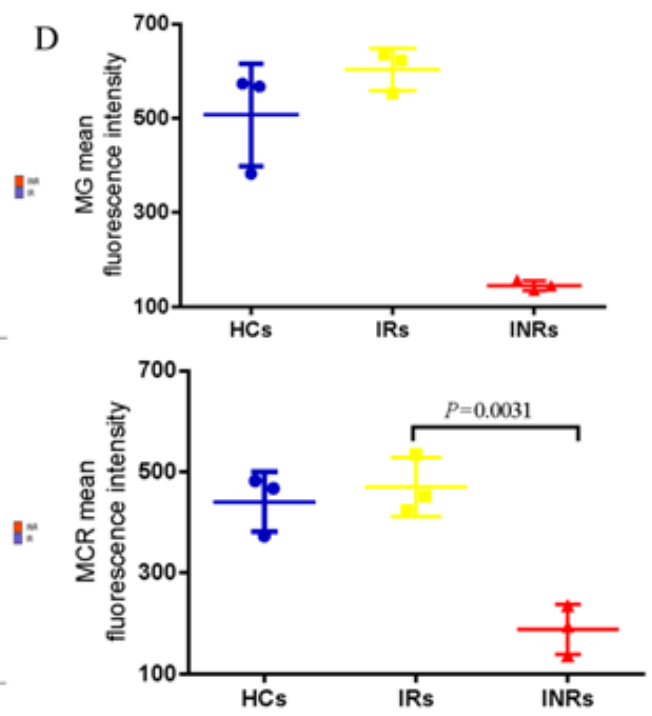

$\mathrm{E}$

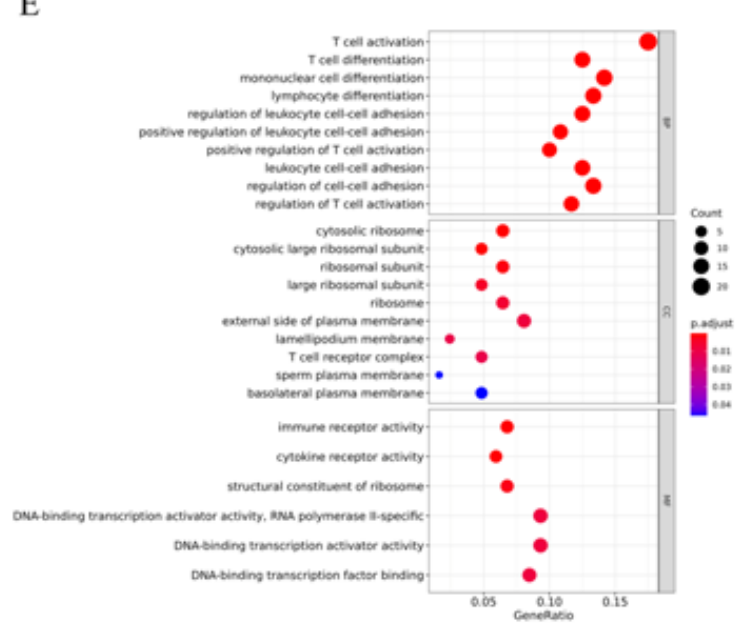

F

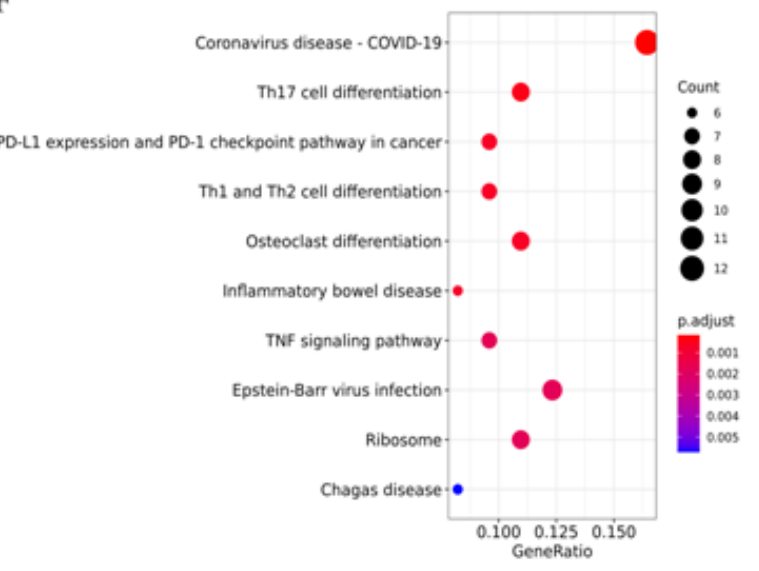

\section{Figure 3}

MAIT cells derived from INR show apoptotic gene expression profile. (A) Heatmaps of differentially expressed genes in MAIT cells between IR and INR groups. (B) Violin plots of the proapoptotic gene expression in MAIT cells from INRs and IRs. (C) Violin plots of mitochondrial function related genes expression in MAIT cells from INRs and IRs. (D) Mitochondrial mass and mitochondrial membrane potential estimated by MG fluorescence and MRC fluorescence in MAIT cells from INRs and IRs, 
respectively. (E, F) GO and KEGG were analyze using DAVID for up-regulated genes in MAIT cells from INRs compared with IRs.
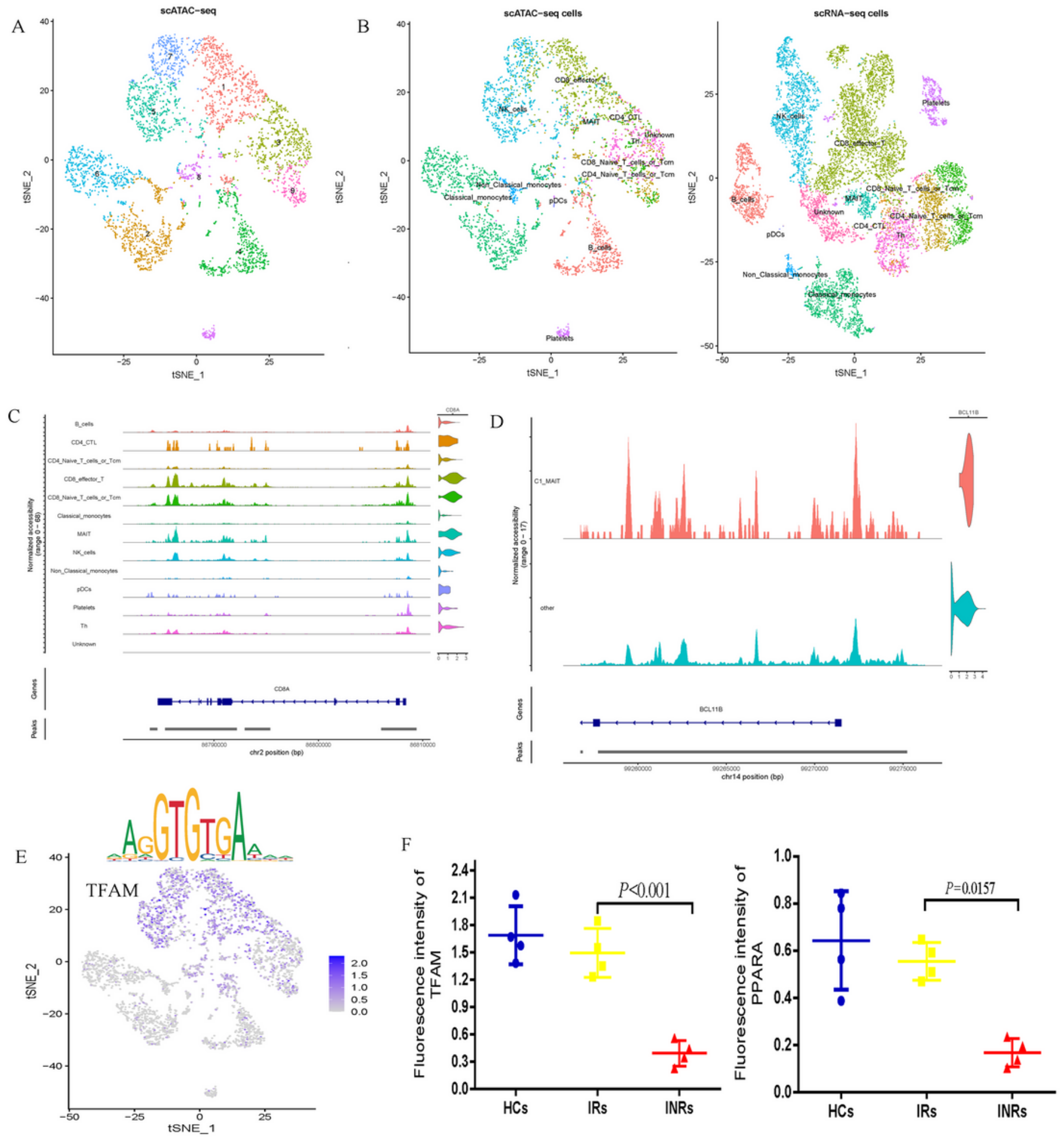

Figure 4

scATAC-Seq Reveals Open Chromatin Landscapes of Single Cells in INR. (A) tSNE plots showing singlecell open chromatin profiles analyzed in the study, color coded for cell clusters. (B) Display after integration of scATAC-Seq and scRNA-seq. (C)scATAC-seq tracks showing open chromatin peaks 
associated with cell-type-specific genes across different cell clusters. (D) Chromatin open state of BCL11B gene in MAIT cells derived from INR. (E) Transcription factor motif enrichment (upper), and gene expression profiles (bottom row) for TFAM. (F) Mean fluorescence intensity of TFAM and PPARA expression in MAIT cells from HCs, IRs and INRs.
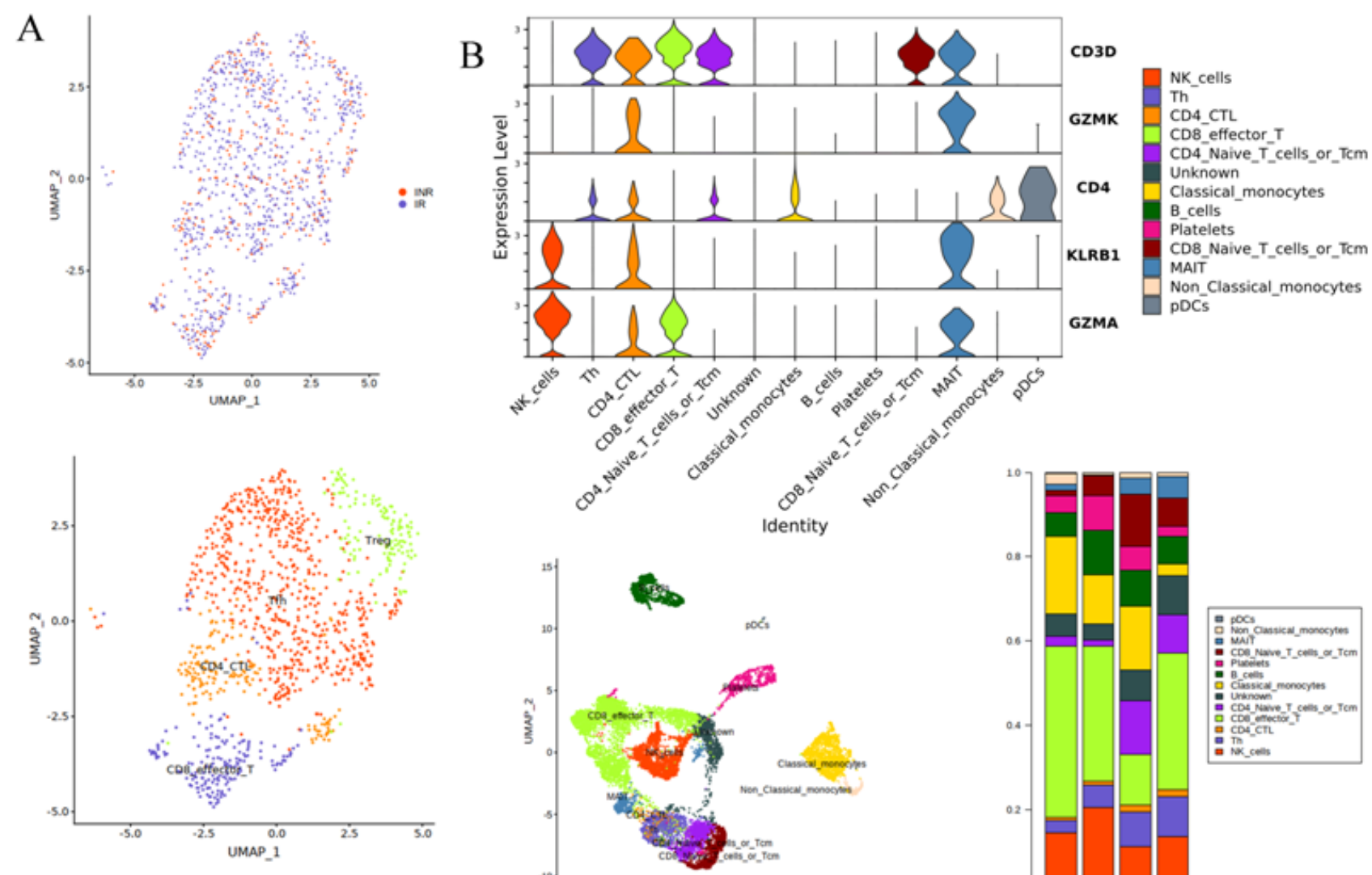

CD8_Naive_T_cells_or_Tcm

MAIT

Non_Classical_monocytes

PDCs
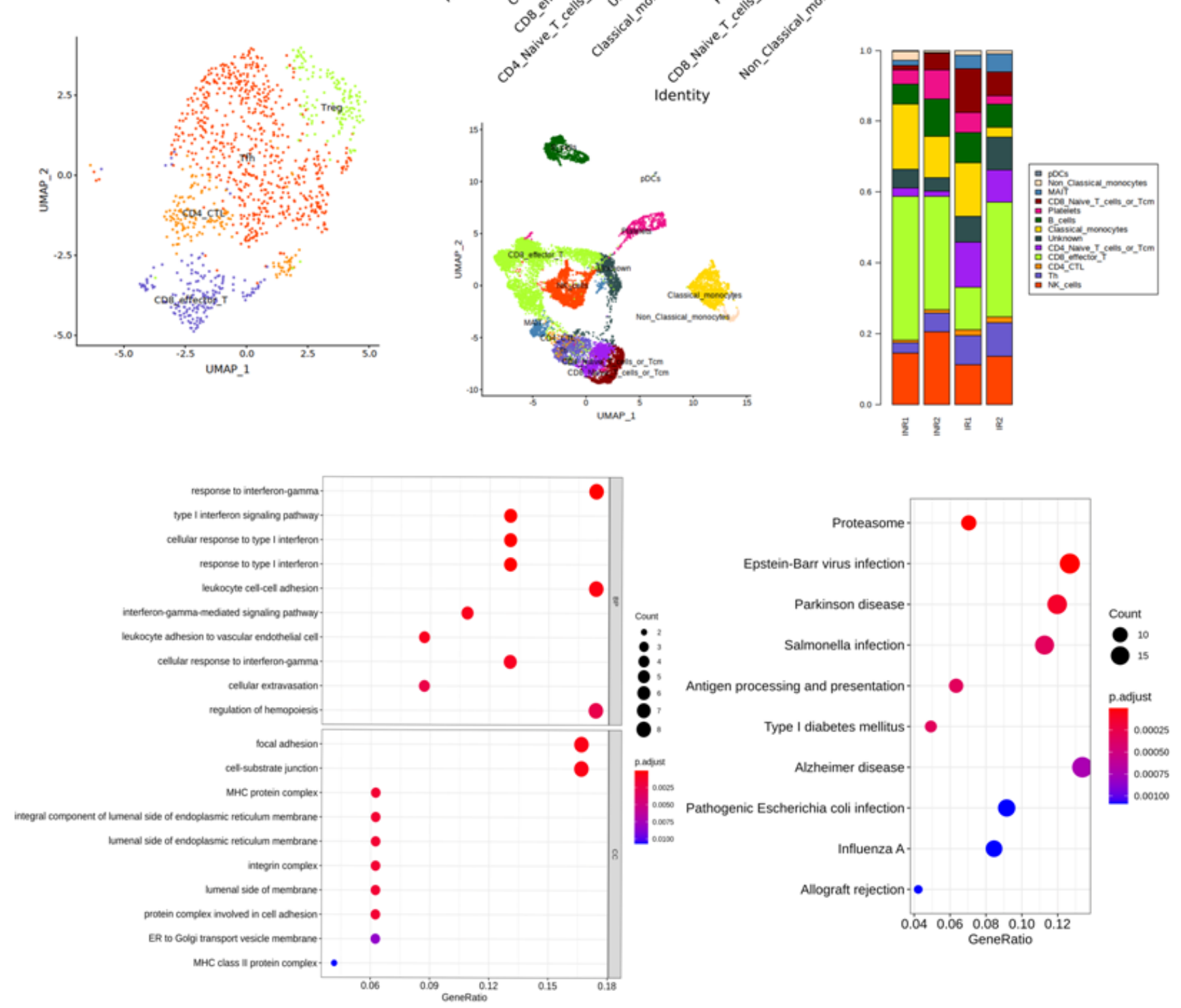

Figure 5 
Profile of Th cells in INRs. (A) Two-dimensional UMAP visualization of Th cells using the Seurat $R$ package. Different colors represent 2 groups (up) Different colors represent different cell subsets (down) (B) Expression and proportion of cytotoxic genes in CD4 CTL. (C) GO and KEGG were analyze using DAVID for up-regulated genes in CD4 CTL cells from INRs compared with IRs.

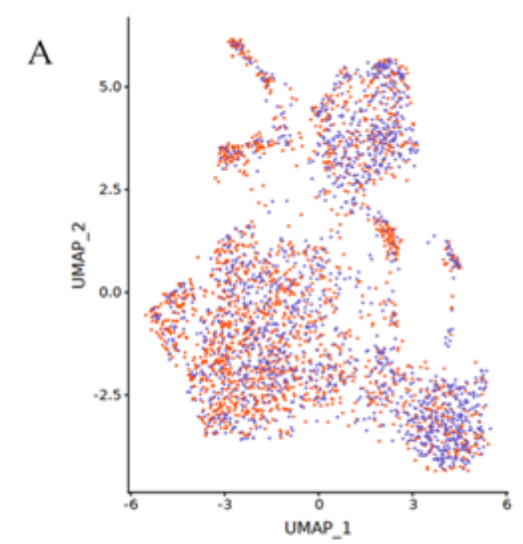

D

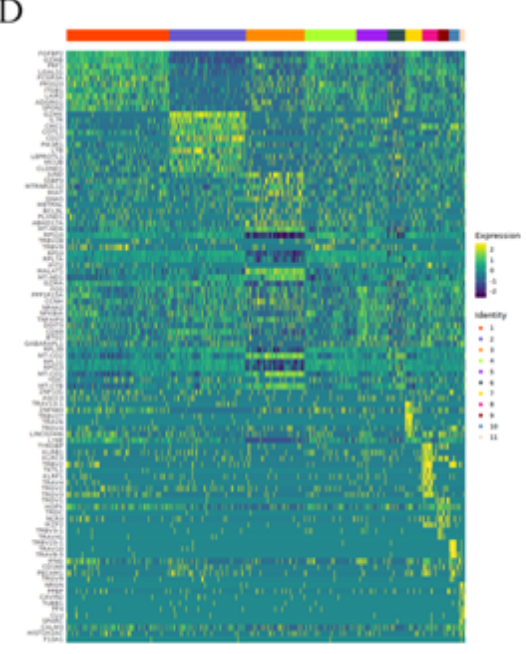

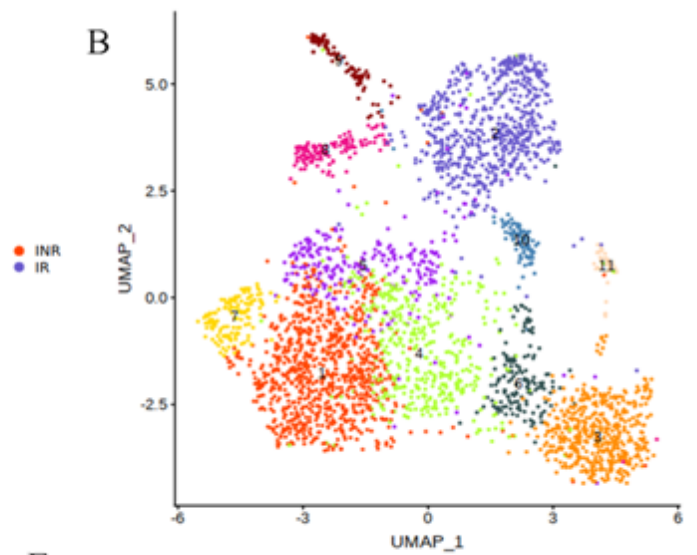

E

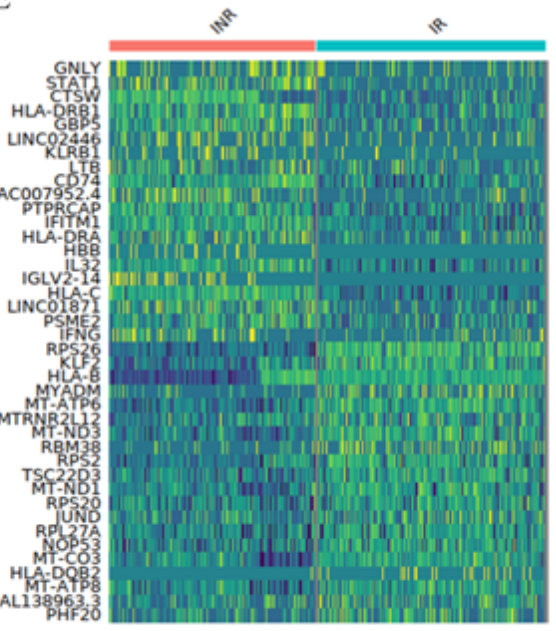

$\mathrm{C}$

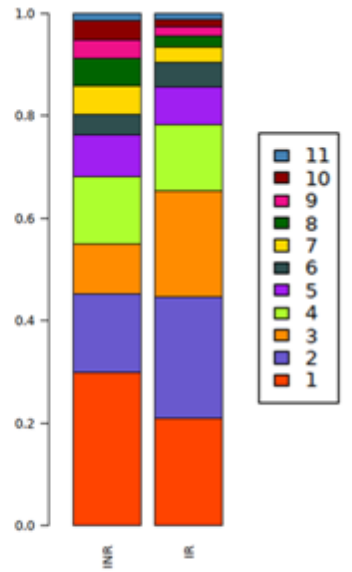

F

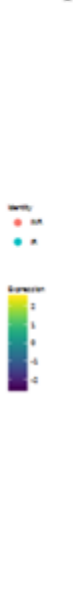

G
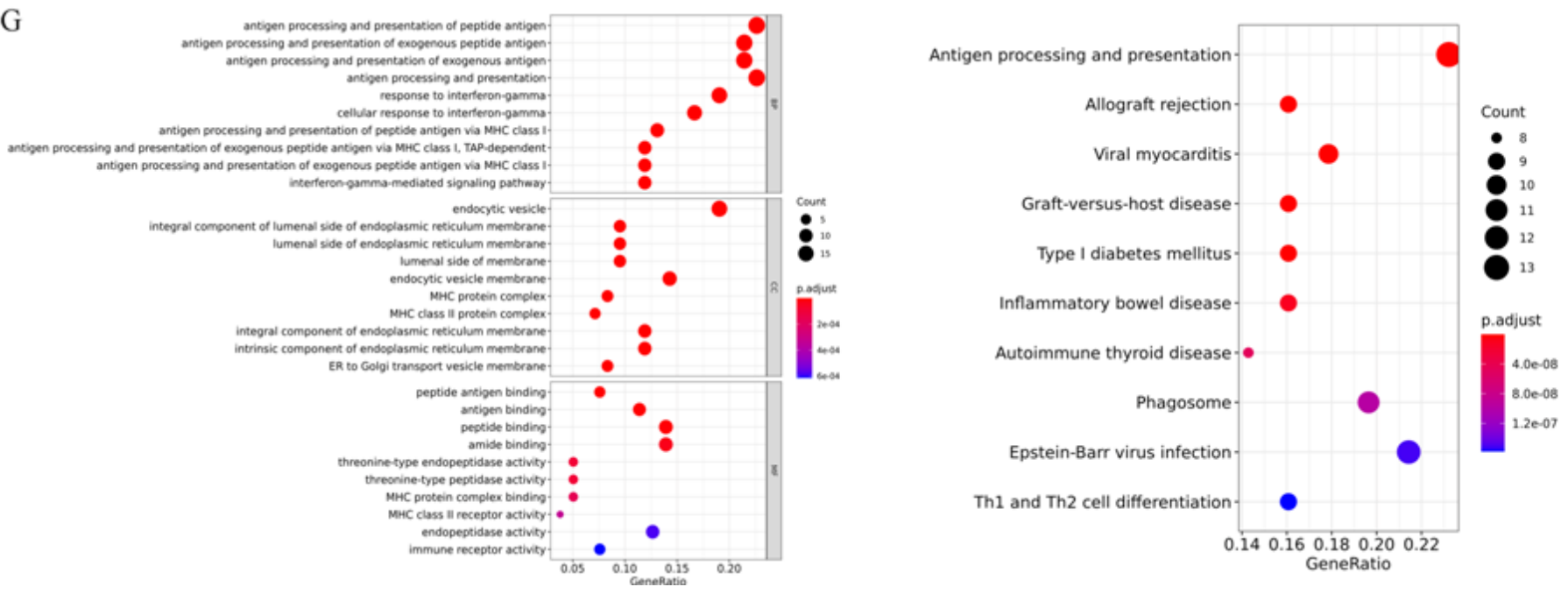

Figure 6

Profile of CD8 effector T cells in INRs. (A) UMAP visualization of CD8 effector T cells using the Seurat R package. Different colors represent 2 groups (left) Different colors represent different cell subsets (right) 
(B) (C) proportion of CD8 effector T cells. (D) significantly expressed genes in CD8 effector T cells between IRs and INRs (E) Heatmap of top 40 genes significantly expressed in CD8 effector T C1(F) Heatmap of top 40 genes significantly expressed in CD8 effector T C2 (G) GO and KEGG were analyze using DAVID for up-regulated genes in CD4 CTL cells from INRs compared with IRs.
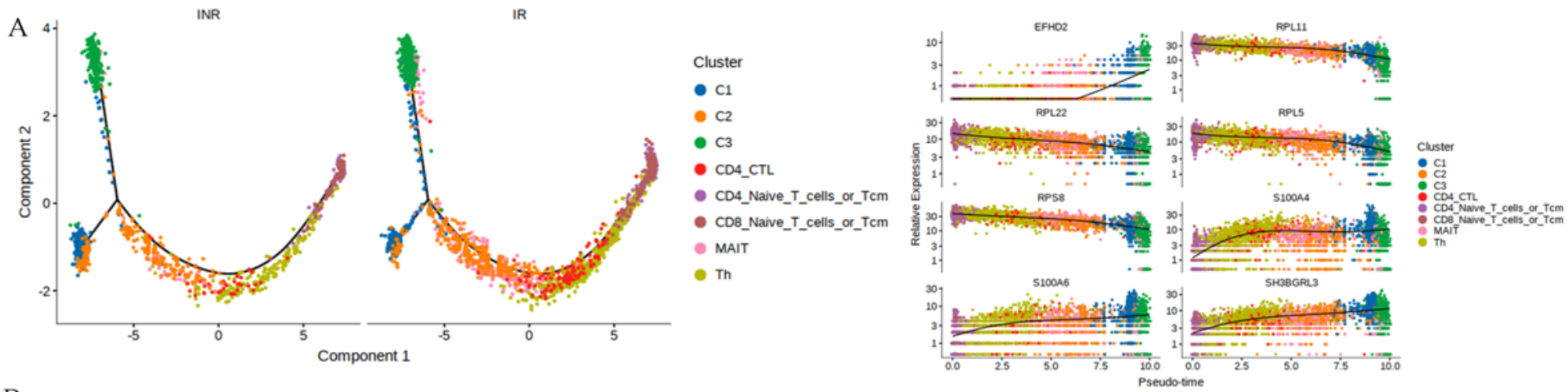

B
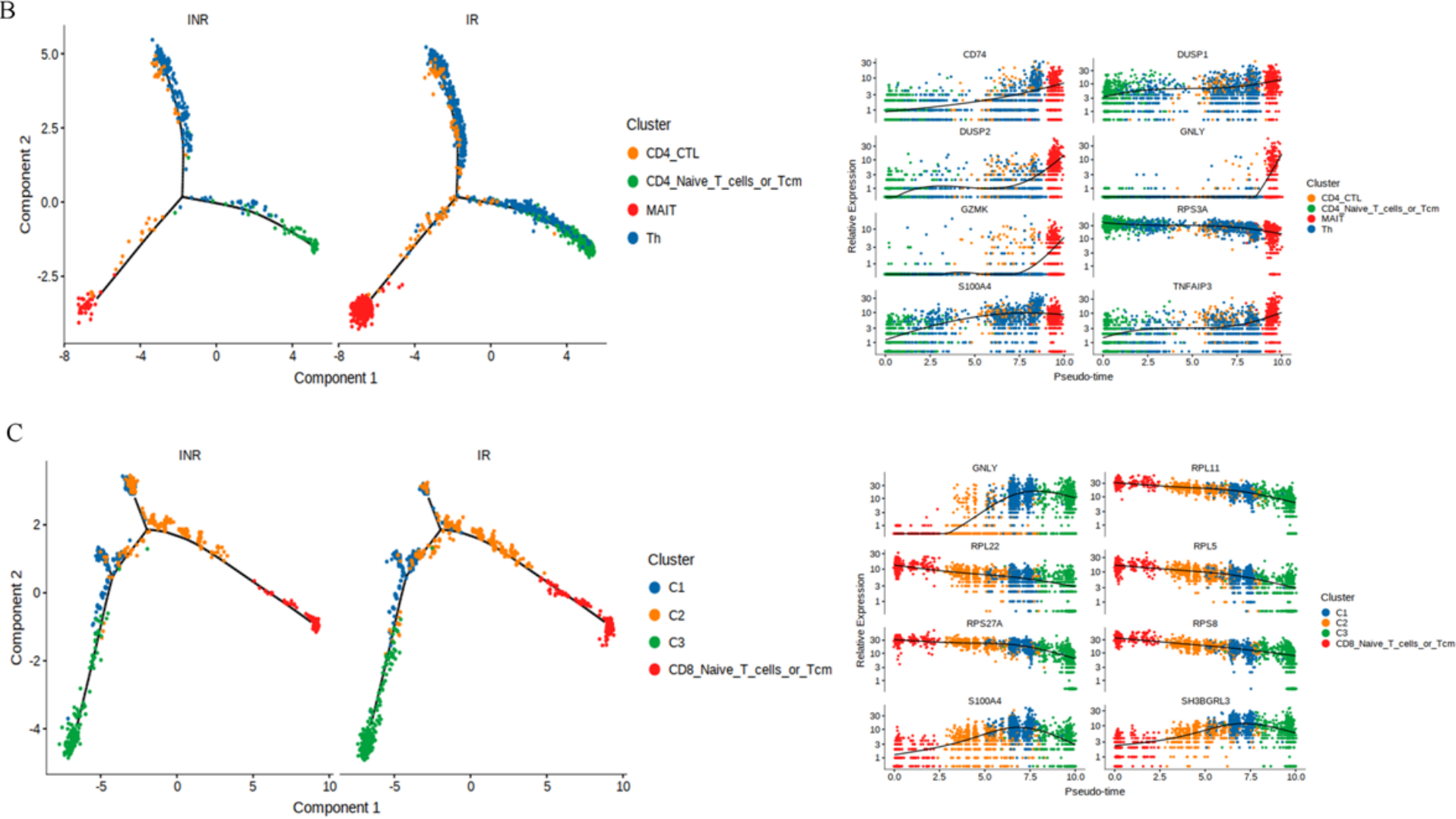

Figure 7

The differentiation state of T cells in INRs and IRs. (A) Pseudotime trajectory of T cells estimated using Monocle 2. A continuous value from 0 to 12 was assigned to each cell as a pseudotime. (Right) Expression transition of differentiation associated genes along the pseudotime. (B) Pseudotime trajectory of CD4 CTL, MAIT and Th cells estimated using Monocle 2. (Right) Expression transition of differentiation associated genes along the pseudotime. (C) Pseudotime trajectory of CD8 effector T cells estimated using Monocle 2. 

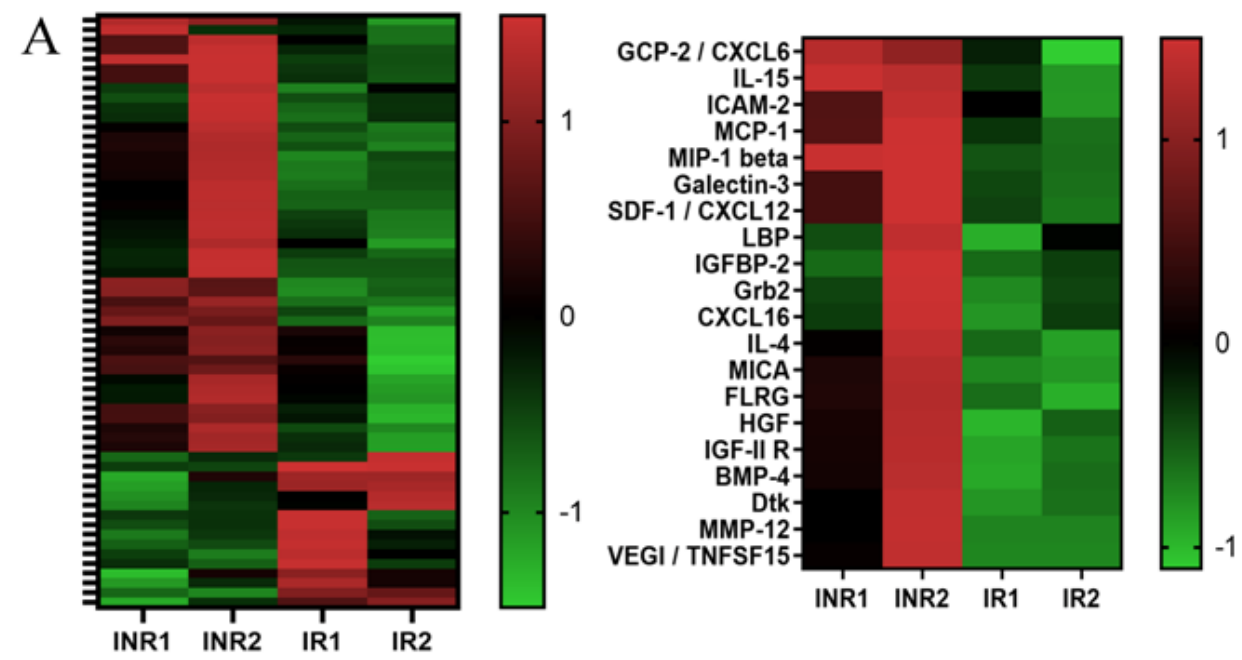

B
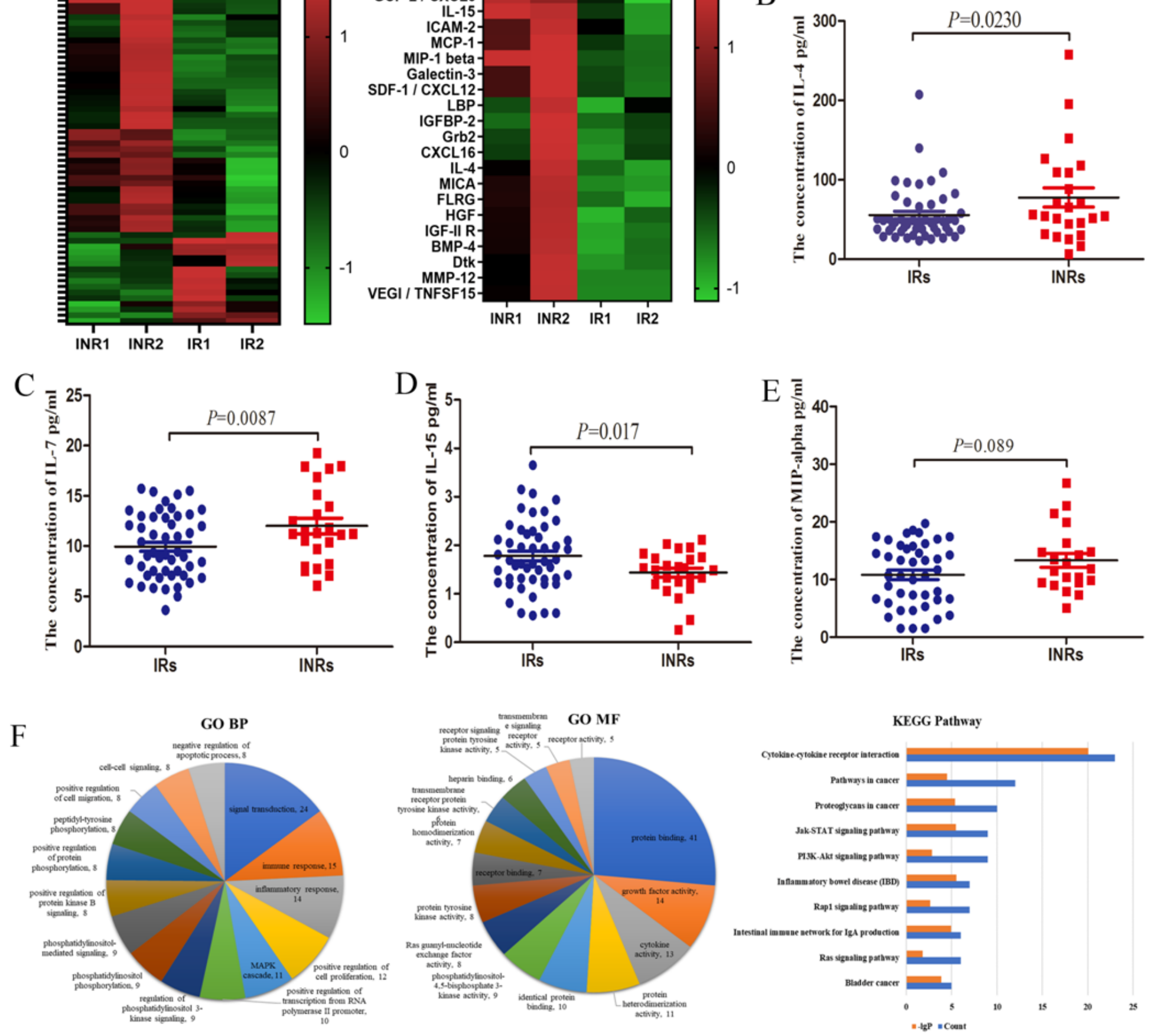

Figure 8

Cytokine profile between INR and IR. (A) Heatmap of significantly expressed cytokine between INRs and IRs using cytokine antibody microarray. (B, C, D and E) Concentrations ( $\mathrm{pg} / \mathrm{ml}$ ) of IL-4, IL-7, IL-15 and MCP-1 in the plasma samples obtained from INR and IR patients. (F) GO and KEGG were analyze using DAVID for up-regulated cytokine in plasma from INRs compared with IRs.

\section{Supplementary Files}


This is a list of supplementary files associated with this preprint. Click to download.

- Supplementaryfigure1.tif

- Supplementaryfigure2.tif 